\title{
Immunohistochemical detection of E7 human papillomavirus protein in pre-malignant and malignant lesions of the uterine cervix
}

\author{
LIJUN SHI, FENGLI HAN, CHENGLONG SHI, YAN HUANG, YAN LIU and XIAOJIA CHANG
}

Attogen Biomedical, Ltd., Suzhou, Jiangsu 215123, P.R. China

Received August 15, 2017; Accepted January 1, 2018

DOI: $10.3892 / \mathrm{mmr} .2018 .9416$

\begin{abstract}
Human papillomavirus (HPV) E7 protein expression is caused by HPV viral DNA integration into human cellular DNA, and is a prerequisite for the development and progression of cervical cancer. The present study aimed to evaluate the role of E7 protein as a biomarker for identification of transformed cervical epithelial cells during the early stages of cervical cancer. Specific monoclonal antibodies to the E7 protein of high-risk HPVs were generated and characterized for applications in immunocytochemistry and immunohistochemistry using cervical epithelial cells or biopsy tissue slides. The specificity and feasibility for detecting precancerous cells in cervical exfoliated epithelial cells was demonstrated. In addition, antibody staining of cervix biopsies indicated the pathological grades of cervical cancer and precancerous lesions. The results of the present study demonstrated the potential benefit of using E7 protein as a novel and specific clinical diagnostic marker to distinguish transient HPV infections from malignant and pre-malignant lesions.
\end{abstract}

\section{Introduction}

Cervical cancer is the second most common malignant tumor in women worldwide, and a leading cause of cancer deaths of females in developing countries (1). Human papillomavirus (HPV) infection is the etiologic agent for virtually all cases of cervical squamous cell carcinoma (SCC) and a large portion of endocervical adenocarcinoma. Among >200 human papillomavirus phenotypes, 14 high-risk HPV (hrHPV) phenotypes have been reported to be closely associated with the initiation and progression of cervical cancer (2). Two genotypes of hrHPV, including HPV 16 and 18, are responsible for $\sim 75 \%$

Correspondence to: Dr Xiaojia Chang, Attogen Biomedical, Ltd., BioBay B3-401, Suzhou Industrial Park, 218 Xinghu Street, Suzhou, Jiangsu 215123, P.R. China

E-mail: xjc2017@attobio.com

Key words: high-risk human papillomavirus, E7 protein, monoclonal antibody, cervical cancer, early detection of all cases of cervical cancer (2). Continuous production of the $\mathrm{E} 7$ protein from oncogenic genotypes of HPV is required for progression of malignancy (2). Thus, sensitive and specific detection of E7 HPV protein expression in the clinical samples of exfoliated epithelial cells or biopsies from cervix may provide a clinical benefit for early detection of precancerous conditions.

The HPV genome consists of six early open reading frames (E1, E2, E4, E5, E6, and E7), two late open reading frames (L1 and L2). Among six proteins encoded by early open reading frames, E6 and E7 are critical for the development of cervical cancer by regulating cervical epithelial cell immortalization (3). Transient viral infection usually resolves spontaneously within 6 to 12 months without increasing the risk of cervical cancer (4). However, in certain cases, viral DNA can integrate into the host genome to cause persistent HPV infection, resulting in an abnormal accumulation of HPV E6 and E7 proteins within host cells (5). Overexpression of E7 protein can competitively bind to the tumor suppressor protein, retinoblastoma, to cause the disassociation of E2F proteins, leading to the abnormal proliferation of cervical cells $(6,7)$ and indispensably contributing to the development of cervical intraepithelial neoplasia (CIN), adenocarcinoma in situ and invasive cervical carcinoma $(4,8)$.

Viral genotyping analysis to identify the oncogenic HPV has not produced good predictive values for the development of intraepithelial CIN lesions (8). It has been reported that $12-14 \%$ of low-grade squamous intraepithelial lesions (LSIL) progress to high-grade squamous intraepithelial lesions (HSIL) (9); without treatment, a few of those lesions progress to invasive cancer (10). Further investigation is required to identify other parameters with clinically meaningful predictive values.

Protein biomarkers involved in the development of cervical cancer, including SCC antigen, serum fragments of cytokeratin, carcinoma embryonic antigen, soluble CD44 and matrix metalloproteinases, have been considered as potential diagnostic markers for cervical cancer screening (11). However, these potential protein biomarkers are encoded by the human genome; therefore, diagnostic use is inhibited by the endogenous expression of these biomarkers within noncancerous cervical cells. The oncoprotein E7 encoded by the hrHPV genome with specific expression in HPV-transformed human cervical cells may serve as an ideal biomarker for the early detection of cervical cancer (12). 
In the present study, investigation of the E7 oncoprotein marker via immunostaining of exfoliated epithelial cells and formalin-fixed paraffin-embedded biopsies for the identification of pre-malignant and malignant lesions in squamous cervical carcinoma was performed.

\section{Materials and methods}

Cervical cancer cell lines. CaSki cell line (HPV16 positive; CRL-1550 ${ }^{\mathrm{TM}}$ ), HeLa cell line (HPV18 positive; CCL-2 ${ }^{\mathrm{TM}}$ ) and C-33A cell line (HPV negative; HTB-31 ${ }^{\mathrm{TM}}$ ) were purchased from the American Type Culture Collection (ATCC; Manassas, VA, USA). CaSki cells were cultured in RPMI-1640 medium (SH30027; Hyclone; GE Healthcare Life Sciences, Logan, UT, USA) containing $10 \%$ heat-inactivated fetal bovine serum (FBS; Gibco; Thermo Fisher Scientific, Inc., Waltham, MA, USA), $100 \mathrm{U} / \mathrm{m}$ penicillin and $100 \mathrm{~g} / \mathrm{ml}$ streptomycin. HeLa and C-33A cells were cultured in Minimum Essential Medium (SH30024; Hyclone; GE Healthcare Life Sciences) containing $10 \%$ heat-inactivated FBS, $100 \mathrm{U} / \mathrm{ml}$ penicillin and $100 \mathrm{~g} / \mathrm{ml}$ streptomycin. For immunocytochemical (ICC) staining, cells ( $3 \times 10^{4} /$ well) were transferred to a $24-$ well plate and were cultured to reach approximately $60-70 \%$ confluency. Then cells were rinsed twice with $10 \mathrm{mM}$ PBS ( $\mathrm{pH}$ 7.4) and fixed in $4 \%$ paraformaldehyde for $15 \mathrm{~min}$ at room temperature. For other assays, adherent cells were harvested following digestion by trypsin (25200; Gibco; Thermo Fisher Scientific, Inc.). Following two washes with PBS, the harvested cells were fixed in $4 \%$ paraformaldehyde for $10 \mathrm{~min}$ at room temperature for liquid based cytology (LBC).

Preparation of $E 7$ recombinant proteins. DNA fragments encoding E7 proteins in hrHPV16, 18, 31, 33, 35, 39, 45, 52, 58 and 59 strains, and low-risk HPV (lrHPV) 6 and 11 strains were cloned and incorporated into pET-21a(+) (Novagen; Merck KGaA, Darmstadt, Germany) or pGEX-4T-1 (GE Healthcare Life Sciences) plasmids, and then were expressed in Escherichia coli (E. coli) BL21/DE3 (American Type Culture Collection, Manassas, VA, USA) to purify His-tagged or glutathione S-transferase-tagged fusion protein. Protein purification was performed as described previously $(13,14)$. The aforementioned proteins tagged with green fluorescent protein were prepared by transient expression in 293 mammalian cell line (ATCC; CRL-3216). Coding sequences of E7 proteins were optimized from original HPV genomic sequences. The accession numbers in Genbank were as follows: AHK23257.1 (HPV16), AGM34461.1 (HPV18), AGM34454.1 (HPV31), AGM34459.1 (HPV33), ACV53985.1 (HPV35), AGU90520.1 (HPV39), AGM34464.1 (HPV45), AET07150.1 (HPV52), AFO63477.1 (HPV58) and ACL12335.1 (HPV59).

Preparation of E7 monoclonal antibodies (mAbs). The present study was approved by the ethics committee of The First Affiliated Hospital of Soochow University (Soochow, China). mAbs against hrHPV E7 proteins were generated by immunizing mice or rabbits with purified HPV16 or 18 recombinant proteins, followed by multistage procedures of established technologies for screening antibody clones from hybridoma (15) and from phage displayed antibody libraries (16-18). Individual clones of mAbs were analyzed by various assays including, ELISA with $E$. coli-derived recombinant hrHPVs and lrHPVs E7 proteins, western blot analysis using E. coli or mammalian-expressed E7 proteins or endogenous E7 proteins from cervical cancer cell lines, ICC using cervical cancer cell lines, and immunohistochemistry (IHC) using human cancer tissue sections. Immunoreactivity of antibodies generated from HPV16 E7 or HPV18 E7 immunized mice or rabbit were tested against E7 proteins from other HPV strains by ELISA using the aforementioned recombinant proteins. Subsequently, mAb clones with desirable properties from either murine or rabbit origins were selected for molecular cloning of immunoglobulin $\mathrm{G}$ ( $\mathrm{IgG}$ ) genes. Cloning of IgG genes was accomplished by isolating the coding sequence of immunoglobulin heavy chain and light chain from hybridoma cells, or scFv sequences in the M13 phage, as described previously (19). The intact IgG molecules for mAb from murine (clone no. E7MuB6 and E7MuH1) and from rabbit (clone no. E7Rb04 and E7Rb19) were produced by large-scale 293 cell transfection using Lipofectamine ${ }^{\circledR}$ LTX reagent (Thermo Fisher Scientific, Inc.) according to manufacturer's instructions. Secreted $\operatorname{IgG}$ protein in culture medium was subjected to affinity purification by chromatography using a Protein-A Sepherose 4B column (cat. no. 101041; Thermo Fisher Scientific, Inc.). Protein concentration of purified IgG was determined by measuring the absorbance at $280 \mathrm{~nm}$ and the purity was analyzed via SDS-PAGE. Immunological activities of antibodies were analyzed by ELISA, and ICC and IHC staining.

The cyclin dependent kinase inhibitor $2 \mathrm{~A}$ (p16 ${ }^{\mathrm{INK} 4 \mathrm{~A}}$; clone no. E6H4; cat. no. 705-4713) antibody was purchased from Ventana Medical Systems, Inc. (Oro Valley, AZ, USA) and secondary antibody reagent EnVision+ Peroxidase system (cat. no. K5007) was from Dako (Agilent Technologies, Inc., Santa Clara, CA, USA).

Specimens. The use of human tissue in the present study was approved by the Institutional Review Board at the First Affiliated Hospital of Soochow University (Soochow, China). Tissue slides (three slides from each sample) used for the evaluation of the IHC staining were prepared using formalin-fixed and paraffin-embedded (FFPE) tissue blocks. Specimens with cervical LSIL (45 samples), HSIL (64 samples) and invasive SCC (7 samples) were selected from the tissue archives in the Pathology Department at the First Affiliated Hospital of Soochow University. The age of the patients ranged from 28-66 years with an mean age of 41.8 years. All specimens were cut into 4-5 mm sections. Three slides from each tissue sample were selected for hematoxylin and eosin staining, E7 antibody immunostaining and $16^{\mathrm{INK} 4 \mathrm{~A}}$ antibody immunostaining.

Exfoliated cervical epithelial cells were obtained from 13 patients with HSIL at the Cancer Hospital at the Chinese Academy of Medical Sciences (Beijing, China). Samples from 10 healthy individuals were obtained from Peking University Hospital (Beijing, China). Cells were preserved in an LBC fixative solution and used within 6 months post-fixation. Those specimens were previously stained in Pap-stain and graded according to the 2001 Bethesda System (20) by the pathologists at Peking University First Hospital and Cancer Hospital Chinese Academy of Medical Sciences (Beijing, China). Slides were treated with a graded series of ethanol 
(100, 70, 50\% and water; $1 \mathrm{~min}$ each), followed by staining with hematoxylin solution, Harris modified (Sigma-Aldrich; Merck $\mathrm{KGaA}$ ) at room temperature for $5 \mathrm{~min}$. Slides were washed with water for $10 \mathrm{sec}$ and incubated with $0.5 \%$ hydrochloric acid for $8 \mathrm{sec}$, followed by a further wash with tap water for $5 \mathrm{~min}$. Subsequently, slides were treated with a graded series of ethanol (50, 70, 80 and 96\%; $30 \mathrm{sec}$ each), followed by EA-50 staining at room temperature for $2.5 \mathrm{~min}$ and washing with $95 \%$ ethanol for $1 \mathrm{~min}$ and $100 \%$ ethanol for 1 min. Finally, slides were washed twice with xylene for 2 min each and mounted with VectaMount ${ }^{\mathrm{TM}}$ Permanent Mounting Medium (Vector Laboratories, Burlingame, CA, USA). The study protocol was proved by Institutional Review Board at the Peking University First Hospital and Cancer Hospital Chinese Academy of Medical Sciences.

Western blotting. Western blot analysis of endogenous E7 proteins was performed by immunoprecipitation of cell lysates. Protein was extracted from CaSki, HeLa and C-33A cervical cancer cell lines using radioimmunoprecipitation lysis and extraction buffer (Thermo Fisher Scientific, Inc.). Immunoprecipitation was performed as described previously (21). Protein concentration was determined with the bicinchoninic acid method. Protein (20 $\mu \mathrm{g} /$ lane) was subjected to $10 \%$ SDS-PAGE, transmembrane and immunoblotting with anti-E7 antibodies. Membranes were blocked at room temperature for $10 \mathrm{~min}$ with TBS-Tween (TBST; $50 \mathrm{mM}$ Tris- $\mathrm{HCl}, 150 \mathrm{mM} \mathrm{NaCl}, 0.5 \%$ Tween $20, \mathrm{pH}$ 7.6) containing $5 \%$ non-fat milk. Following washing, membranes were incubated with the aforementioned primary monoclonal E7 antibodies $(1: 1,000)$ overnight at $4^{\circ} \mathrm{C}$. Following washing, membranes were incubated with the horseradish peroxidase (HRP)-labeled goat anti-mouse secondary antibody (1:1,000; cat. no. A2554, Sigma-Aldrich; Merck KGaA, Darmstadt, Germany) or HRP-labeled mouse anti-rabbit secondary antibody (1:1,000; cat. no. A1949, Sigma-Aldrich; Merck KGaA) at room temperature for $2 \mathrm{~h}$. Finally, signals were detected by adding HRP substrate solution containing 3,3'-diaminobenzidine chromogen (cat. no. ZLT-9033; OriGene Technologies, Inc., Beijing, China).

ICC. For ICC analysis, the prepared Pap-smear slides and LBC (ThinPrep ${ }^{\circledR}$; Hologic China, Inc., Beijing, China) slides prepared according to the manufacturer's protocols were immediately fixed in $99 \%$ ethanol at room temperature for $1 \mathrm{~h}$, and were air-dried at room temperature overnight. Prior to antibody staining, slides were rehydrated in $50 \%$ ethanol for $10 \mathrm{~min}$ and distilled water for $\geq 30 \mathrm{sec}$. Antigen retrieval was performed by heat treatment in epitope retrieval buffer (Tris-EDTA, pH 9.0) at $95-99^{\circ} \mathrm{C}$ for $10 \mathrm{~min}$ and then slides in epitope retrieval buffer were maintained at room temperature for 20 min until the temperature decreased below $50^{\circ} \mathrm{C}$. Cells were fixed in $4 \%$ paraformaldehyde at room temperature for $2 \mathrm{~h}$. and permeabilized in $0.3 \%$ Triton X-100 for $15 \mathrm{~min}$ at room temperature, followed by washing twice with TBST. ICC staining was then conducted. Briefly, slides were rinsed once in TBST and treated with $3 \% \mathrm{H}_{2} \mathrm{O}_{2}$ at room temperature for 10 min to block endogenous peroxidase. Slides were blocked in $10 \%$ newborn bovine serum (Thermo Fisher Scientific, Inc.) at room temperature for $1 \mathrm{~h}$, followed by incubation with primary E7 antibody, E7MuB6 or E7MuH1 $(1: 1,000)$ at room temperature $\left(20-25^{\circ} \mathrm{C}\right)$ for $4 \mathrm{~h}$. Slides were subsequently treated with secondary antibody reagent EnVision ${ }^{+}$Detection Systems Peroxidase/DAB, Rabbit/Mouse (K5007; Dako; Agilent Technologies, Inc.) according to the manufacturer's protocols. Finally, slides were stained with hematoxylin $(0.5 \%)$ at room temperature for $2 \mathrm{~h}$, and dehydrated in a series of graded ethanol concentrations $(75,85,95$ and $100 \%$; $10 \mathrm{~min}$ each) at room temperature. Finally, slides were mounted with coverslips for observation under an optical microscope (Olympus Corporation, Tokyo, Japan). Positive cervical cancer cells revealed staining of the cytoplasm. CaSki was used as the positive control, and C-33A was used as the negative control.

IHC. IHC staining with anti-E7 rabbit mAbs (clone nos. E7R04 and E7R19) or with anti-p16 ${ }^{\mathrm{INK} 4 \mathrm{~A}}$ antibody (clone no. E6H4; cat. no. 705-4713; Ventana Medical Systems, Inc.) were performed on tissue sections. Briefly, slides carrying cervical tissue sections were dewaxed in xylene for $5 \mathrm{~min}$ and rehydrated in a descending ethanol gradient at room temperature $(95,85$, 50 and $30 \%$; 2 min each). Epitope retrieval was performed by heating the slides at $121^{\circ} \mathrm{C}$ for $2 \mathrm{~min}$ in citrate buffer $(10 \mathrm{mmol} / \mathrm{l}$, $\mathrm{pH}$ 6.0). Endogenous peroxidase activity was blocked with treatment with $3 \% \mathrm{H}_{2} \mathrm{O}_{2}$ solution at room temperature for 20 min. Blocking was performed by incubation with TBST containing $10 \%$ newborn bovine serum at room temperature for $1 \mathrm{~h}$. Following washing, primary antibodies $(1: 1,000)$ were incubated with the slides at $20-25^{\circ} \mathrm{C}$ for $1 \mathrm{~h}$, followed by washing with TBST and incubation with HRP-labeled secondary antibody (Dako EnVision+ Peroxidase system) at room temperature for $30 \mathrm{~min}$. DAB chromogen system (Dako; Agilent Technologies, Inc.) was used for color development according to the manufacturer's protocols. Slides were washed with xylene three times, for 3 min each time. Subsequently, slides were treated with a series of graded ethanol $(100,80$, 50 and $30 \% ; 2$ min each) at room temperature. Then slides were stained with hematoxylin $(0.5 \%)$ at room temperature for $20 \mathrm{~min}$. Slides were washed with tap water for $5 \mathrm{~min}$ and then treated with acid alcohol $(1 \% \mathrm{HCl}$ in $70 \% \mathrm{EtOH})$ until the slides turned pink. Following a further wash with tap water for $5 \mathrm{~min}$, slides were treated with ammonia water $(1 \mathrm{ml}$ $\mathrm{NH}_{4} \mathrm{OH}$ in $11 \mathrm{H}_{2} \mathrm{O}$ ) until the sections noticeably darkened. Slides were washed again with tap water for $5 \mathrm{~min}$ and stained with Eosin Y $(0.5 \%)$ at room temperature for $1 \mathrm{~min}$. Following rinsing in tap water for $1 \mathrm{~min}$, slides were dehydrated in a graded ethanol series (50, 80, 90 and 100\%; 2 min each) and mounted on coverslips. The HPV-positive cervical cancer tissue was used as a positive control, and sham-treated slide treated with blank blocking buffer instead of primary antibody was used as negative control.

Interpretation of E7 and p16 $6^{I N K 4 A}$ immunostaining. Stained slides were analyzed by two pathologists for variable representation of epithelial and stromal components and immunoreactivity. Squamous epithelial cells, with brown-colored cytoplasmic staining for E7 protein, or with nuclear and/or cytoplasmic staining for $\mathrm{p} 16^{\mathrm{INK} 4 \mathrm{~A}}$ protein, were identified and graded as follows: 0, no staining; 1, 1-25\% staining; 2, 26-50\% staining; 3, 51-75\% staining; and 4, 76-100\% staining. Cases

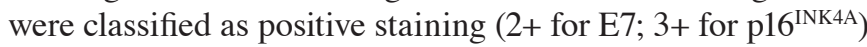


vs. negative or focal staining. The staining intensity for each marker was not taken into consideration. The examination and scoring of immunohistochemistry slides were confirmed by a panel of more than two certified professionals in pathology.

In situ hybridization (ISH) analysis for HPV E7 mRNA. ISH analysis for HPV E7 mRNA was performed using RNAscope HPV kit (Advanced Cell Diagnostics, Inc., Newark, CA, USA) according to the manufacturer's protocols. Briefly, FFPE tissue sections ( $4 \mathrm{~mm}$-thick) were heated $\left(65^{\circ} \mathrm{C}\right)$ for $5 \mathrm{~min}$ and pretreated with protease $\mathrm{K}(10 \mu \mathrm{g} / \mathrm{ml})$ at $37^{\circ} \mathrm{C}$ for $20 \mathrm{~min}$ prior to hybridization. For each case, three slides from adjacent tissue sections were separately hybridized with probes targeting ubiquitin C (cat. no. 310041; Advanced Cell Diagnostics, Inc.), DapB (cat. no. 310043; Advanced Cell Diagnostics, Inc.) and a cocktail of 18 genotypes of hrHPV $(16,18,26,31,33,35,39$, 45, 51, 52, 53, 56, 58, 59, 66, 68, 73 and 82; cat. no. 312591). A HRP-based signal amplification system was subsequently used to hybridize the target probes using a peroxidase activity assay kit (Sigma-Aldrich), and signal generation followed by incubation with the chromogenic substrate 3,3'-diaminobenzidine $(0.05 \%)$ at room temperature for $30 \mathrm{~min}$. Counter staining with hematoxylin was performed using the method described above. A ubiquitin $\mathrm{C}$ probe was used as an endogenous control for RNA integrity assessment. The bacterial gene DapB was used as a negative control to assess background staining. A positive stain was defined by a typical pattern of punctate brown colored granules (precipitates) in or around the nucleus, or in the cytoplasm of the cells. For the paired IHC analysis, adjacent tissue sections were processed by staining with the aforementioned anti-E7 antibody cocktail.

\section{Results}

E7 $m A$ b recognizes endogenous E7 protein in cervical cancer cells. mAbs with high specificity towards E7 recombinant proteins from the hrHPV strains (HPV16, 18, 31, 33, 35, 39, 45, 52, 58 and 59), and without cross-reactivity to E7 proteins from the lrHPV strains (HPV6 and 11) were selected via ELISA. Positive antibody clones were further characterized by their abilities to bind the endogenous E7 protein in cervical cancer cells. As presented in Fig. 1, antibodies immunoprecipitated the endogenous E7 protein from cell lysates prepared from cervical cancer cell lines CaSki (HPV16 positive) and HeLa (HPV18 positive). The C-33A cell lysate was included in the parallel experiment as a negative control. Protein samples from immunoprecipitation were analyzed by western blotting. A single specific band with expected molecular weight was detected in CaSki cell lysate (Fig. 1A) and in HeLa cell lysate (Fig. 1B), indicating that mAbs may specifically recognize endogenous E7 proteins expressed in cancer cell lines. Two upper bands in each lane with apparent molecular weight of 55 and $28 \mathrm{kDa}$ respectively represented the $\mathrm{IgG}$ heavy chain and light chain of $\mathrm{mAb}$ used in the immunoprecipitation.

E7 mAb specifically stained cervical cells by ICC. For further confirmation of the specificity of mAbs against E7 proteins expressed in cervical cells, three cervical cancer cell lines C-33A, HeLa and CaSki were cultured and analyzed via ICC (Fig. 2). HeLa cells exhibited high expression of HPV18
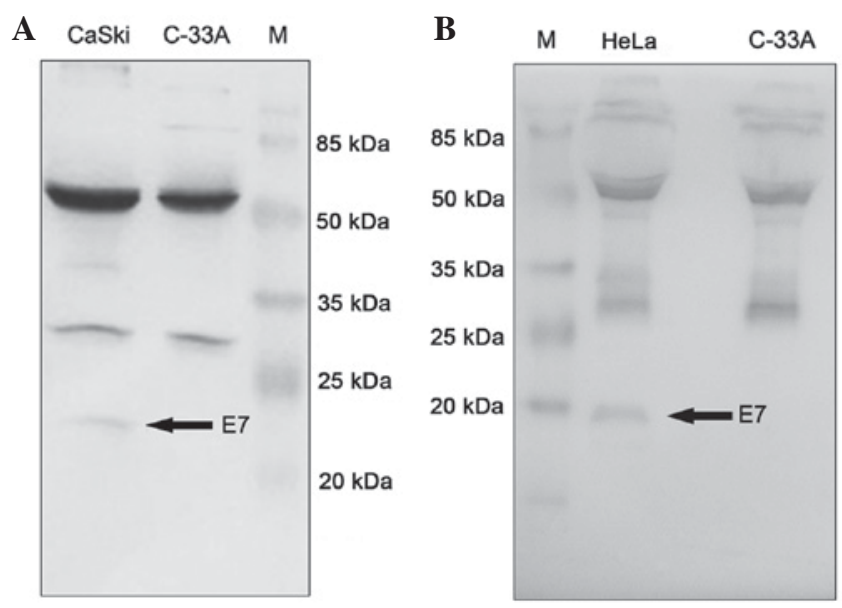

Figure 1. Western blot analyses for the specificity of anti-E7 monoclonal antibodies. E7 proteins in cancer cell lysates were immunoprecipitated and immunoblotted with murine monoclonal antibodies. (A) E7MuB6 protein of HPV16 origin in (A) CaSki and (B) E7MuH1 protein of HPV18 in HeLa were marked with arrows with an apparent molecular weight of $20 \mathrm{kDa}$. C-33A was the negative control used in parallel experiments. The upper bands in each lane with apparent demonstrated molecular weights of 55 and $28 \mathrm{kDa}$ are the heavy and light chains of the monoclonal antibodies used in the immunoprecipitation, respectively. M, protein molecular weight ladder. HPV, human papillomavirus.

\section{A}

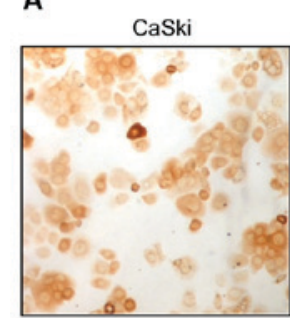

B
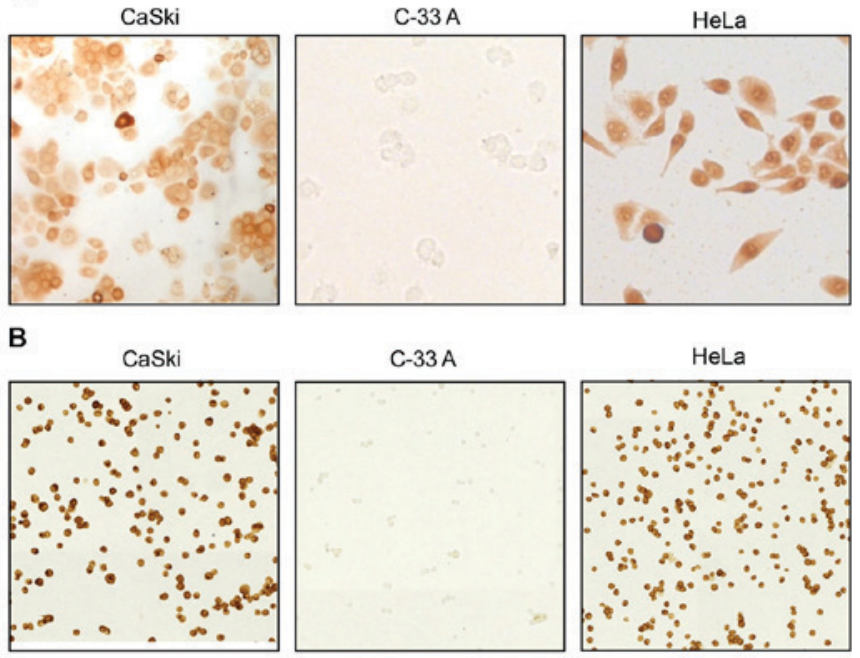

Figure 2. Analyses of the specificity of E7 monoclonal antibodies in cervical cancer cell lines by immunocytochemistry. (A) Intense staining was observed in the cytoplasm of HPV-positive CaSki cells with E7MuB6 antibody in adherent cells (left panel), no staining was observed within the HPV-negative C-33A cells (middle panel); E7MuH1 revealed cytoplasmic and nuclear staining within adherent HeLa cells (right panel). (B) Antibody E7MuH1 stained suspended CaSki cells (left panel), no staining with antibody within the HPV-negative C-33A cells (middle panel), E7MuH1 staining in suspended HeLa cells (right panel). Magnification, x20.

E7 protein, while CaSki cells demonstrated overexpression of HPV16 E7 proteins. C-33A cells were used as a negative control as HPV16 E7 and HPV18 E7 proteins are not expressed within these cells. HeLa cells were exclusively stained by $\mathrm{mAb}$ E7MuH1 against HPV18 E7 protein, while HPV16 E7 protein was specifically detected in CaSki cells using mAb E7MuB6 against HPV16 E7 proteins; neither antibody recognized C-33A cells. Collectively, the results of the present study indicated 
A
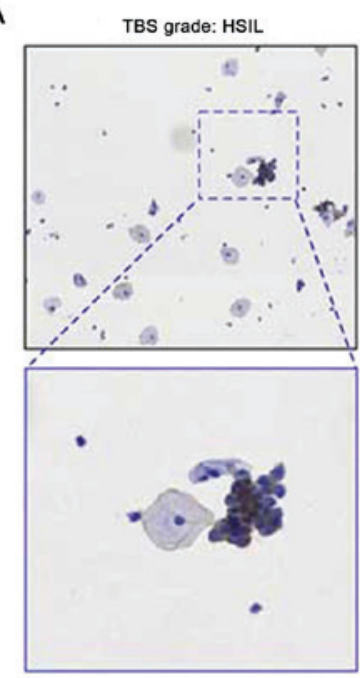

B
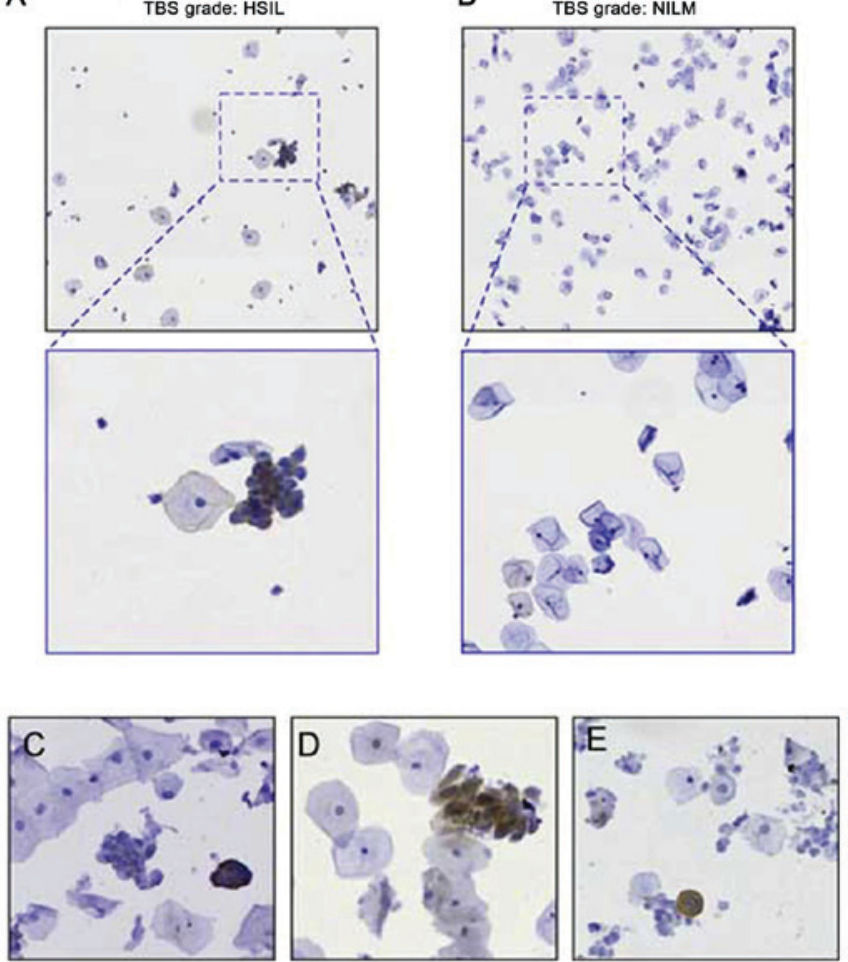

Figure 3. Representative immunocytochemistry staining results of squamous intraepithelial cells using anti-HPV E7 mAbs. Anti-E7 mAb-stained neoplasia cells were observed in (A) HSIL, but not in (B) NILM. Positive staining of E7 in various lesions of dysplastic cells. TBS grade (C) HSIL, (D) low-grade squamous intraepithelial lesion and (E) atypical squamous cells of undetermined significance. mAb, monoclonal antibody; TBS, 2001 Bethesda System; HSIL, high-grade squamous intraepithelial lesion; NILM, negative for intraepithelial lesion and malignancy.

that the mAbs generated have high specificity for E7 proteins expressed in cervical cancer cells.

Application of $m A b$ in ICC staining of exfoliated cervical epithelial cells. LBC or thin-layer cytology has been widely applied in clinical testing for the examination of exfoliated cervical epithelial cells. To investigate the compatibility of E7 $\mathrm{mAb}$ for immunostaining on LBC slides, fixed cervical epithelial cells were treated with mAb E7MuB6. Antibody activity and specificity were demonstrated using positive samples of HSIL and positive HPV16 genotyping results. Normal cervical cell slides negative for intraepithelial lesion and malignancy (NILM) were used as negative controls. As presented in Fig. 3, the slides treated with murine mAb revealed dark brown colored cells, indicating E7 positive expression within the transformed cells, consistent with their HSIL cytological morphology features (Fig. 3A). None of cells on the slides NILM were stained by the E7 mAb (Fig. 3B). In addition, LBC slides with varying lesion grades [HSIL (Fig. 3C), LSIL (Fig. 3D), and atypical squamous cells of undetermined significance (ASC-US; Fig. 3E)] could be identified via a direct evaluation based on the expression of the oncoprotein E7. This demonstrated the feasibility of using a specific biomarker for the interpretation of the cytopathological results. As presented in Fig. 3E, a case classed as ASC-US case by the conventional cytology test was identified as positive for precancerous cells based on the identification of E7 oncoprotein expression.
A
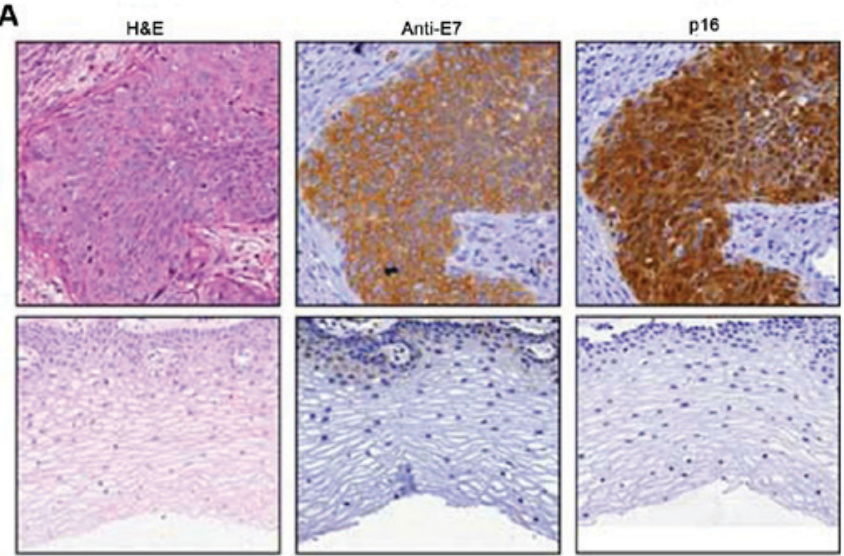

B
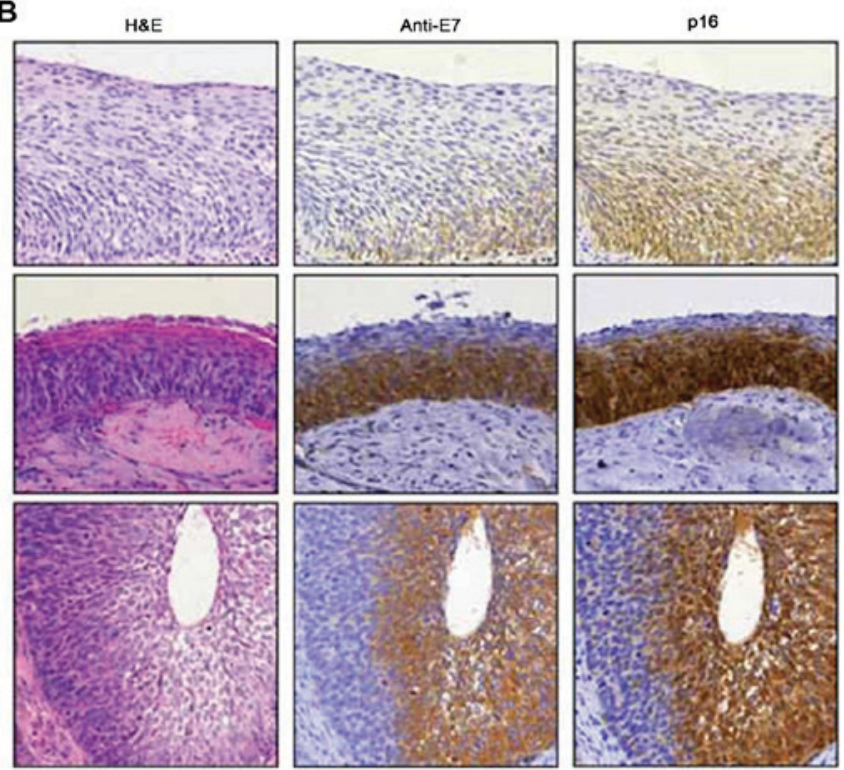

Figure 4. Representative results of immunohistochemistry staining with E7 $\mathrm{mAbs}$ and $\mathrm{p} 16^{\mathrm{INK} 4 \mathrm{~A}}$ antibody. (A) Cervical carcinoma lesions (top panels) and normal cervical tissues (bottom panels) were stained with H\&E, E7 $\mathrm{mAbs}$ and $\mathrm{p} 16^{\mathrm{INK} 4 \mathrm{~A}}$ antibody (left to right). (B) Representative CIN1 (top panels), CIN2 (middle panels) and CIN3 (bottom panels) lesions were stained with $\mathrm{H} \& \mathrm{E}, \mathrm{E} 7 \mathrm{mAbs}$ and $\mathrm{p} 16^{\mathrm{INK} 4 \mathrm{~A}}$ antibody. Magnification, x20. mAbs, monoclonal antibodies; CIN, cervical intraepithelial neoplasia; H\&E, hematoxylin and eosin; $16^{1 \mathrm{NK} 4 \mathrm{~A}}$, cyclin dependent kinase inhibitor $2 \mathrm{~A}$.

$I H C$ reactivity with $E 7$ protein and comparison with surrogate p16 INK4A marker. Pathology tests are considered to be the most important test for the clinical diagnosis of cervical cancer or precancerous lesions. To evaluate the utility of E7 antibodies for histopathological examination of cervical cancer, the IHC staining capacity of the rabbit monoclonal anti-E7 cocktail was investigated and compared with the anti-E7 immunostaining patterns of the established surrogate protein marker $\mathrm{p} 16^{\mathrm{INK} 4 \mathrm{~A}}$. As presented in Fig. 4A, rabbit monoclonal anti-E7 mAbs exhibited intense granular staining in cytoplasm of human cervical carcinoma lesions (top), while no brown staining in normal cervical tissues was observed (bottom). In addition, E7 mAbs demonstrated a similar staining pattern to that of anti-p16 ${ }^{\mathrm{INK} 4 \mathrm{~A}}$ antibody in different premalignant and malignant lesions (Fig. 4A and B).

It has been speculated that the tumorigenic functions of E7 protein may be associated with its expression levels during the development and progression of cancer. To further investigate whether the E7 antibodies used in the present study may reveal 
Table I. E7 and p16 ${ }^{\mathrm{INK} 4 \mathrm{~A}}$ immunostaining in tissue slides via immunohistochemistry.

\begin{tabular}{|c|c|c|c|c|c|c|}
\hline \multirow[b]{2}{*}{ Protein expression } & \multicolumn{2}{|c|}{ LSIL } & \multicolumn{2}{|c|}{ HSIL } & \multicolumn{2}{|c|}{ SCC } \\
\hline & Cases & $\%$ & Cases & $\%$ & Cases & $\%$ \\
\hline \multicolumn{7}{|l|}{ E7 } \\
\hline Negative & 31 & 68.9 & 6 & 9.4 & 0 & 0 \\
\hline Positive & 14 & 31.1 & 58 & 90.6 & 7 & 100 \\
\hline Total & 45 & & 64 & & 7 & \\
\hline \multicolumn{7}{|l|}{$\mathrm{p} 16^{\mathrm{INK} 4 \mathrm{~A}}$} \\
\hline Negative & 35 & 77.8 & 4 & 6.3 & 0 & 0 \\
\hline Positive & 10 & 22.2 & 60 & 93.7 & 7 & 100 \\
\hline Total & 45 & & 64 & & 7 & \\
\hline
\end{tabular}

p16 ${ }^{\mathrm{INK} 4 \mathrm{~A}}$, cyclin dependent kinase inhibitor 2A; HSIL, high grade squamous intraepithelial lesions; LSIL, low grade squamous intraepithelial lesions; SCC, squamous cell carcinoma.

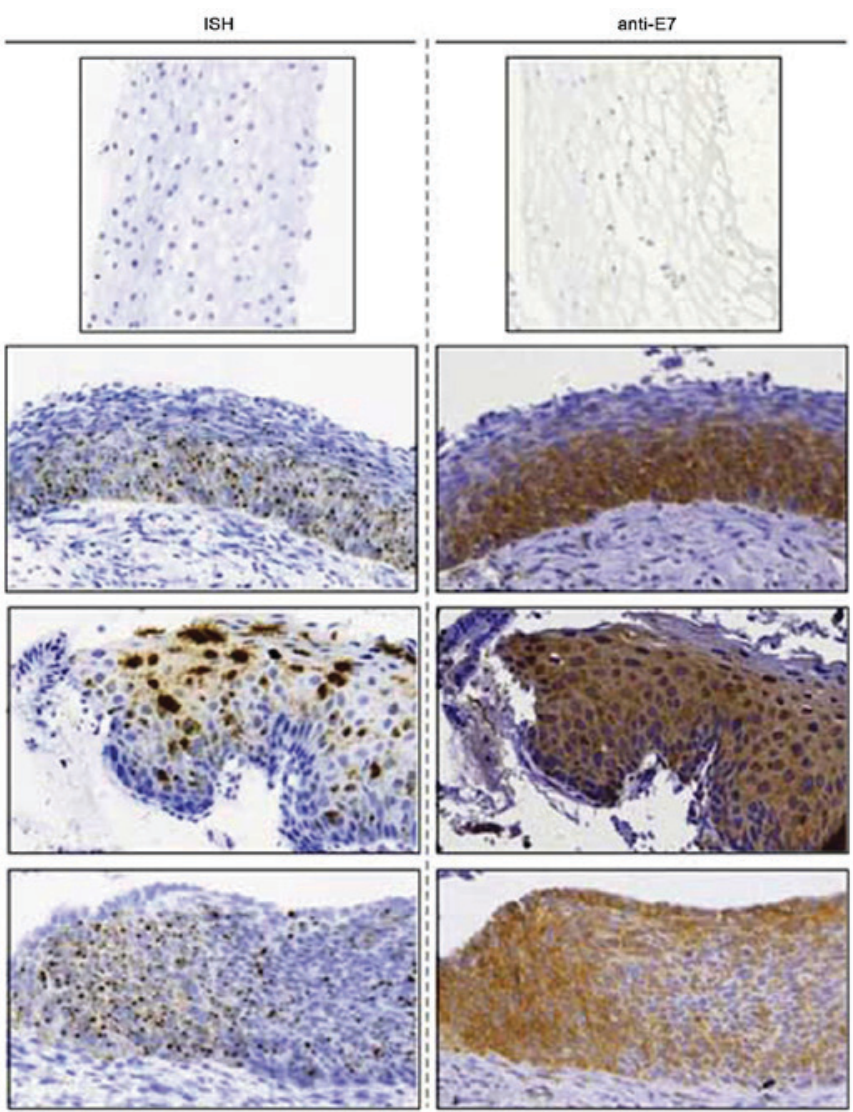

Figure 5. Representative results of ISH and IHC staining of cervical carcinoma lesion. Adjacent sections of high-grade squamous intraepithelial lesion tissue were analyzed via ISH with E7 mRNA probes (left panels) or IHC with E7 monoclonal antibodies cocktail (right panels). Top panel demonstrated the negative controls with normal cervix tissue in both assays. Magnification, x20. IHC, immunohistochemistry; ISH, in situ hybridization.

the expression levels of HPV E7 proteins in cervical lesions of increasing pathological grades, tissue sections from cervical intraepithelial neoplasia (CIN)1, CIN2 and CIN3 samples were subjected to IHC staining using the novel rabbit anti-E7 mAbs (Fig. 4B). The staining results indicated an increased amount of E7 positive cells on the slides as the pathological stage was increased. This observation of E7 staining is consistent with the increased severity of pathological stages, from CIN1 to CIN2, and then to CIN3 lesions. Comparison of the E7 and p16 ${ }^{\text {INK4A }}$ antibody staining results in normal tissue (negative) and cervical carcinoma (positive) demonstrated similar staining patterns as presented in Fig. 4A. A notable difference is that the $16^{\mathrm{INK} 4 \mathrm{~A}}$ antibody stained numerous foci in morphologically normal cervical cells, whereas the E7 antibody demonstrated an overall similar staining pattern, but with a distinct specificity to the cervical cancer lesions (Fig. 4B).

To assess the robustness of the E7 immunostaining in a larger number of pathology specimens, an antibody reagent cocktail of anti-E7 mAbs was optimized and used to test tissue slides from 116 cases with pathology reports in a double-blind experiment. For comparison, p16 $^{\mathrm{INK} 4 \mathrm{~A}}$ immunostaining was performed in parallel experiments. The results were analyzed against the gold standard of pathology. As presented in Table I, the staining of E7 among the three pathological groups, LSIL, HSIL and SCC exhibited a trend of increasing E7-positive rates: 31.1, 90.6 and $100 \%$, respectively. The p16 ${ }^{\mathrm{INK} 4 \mathrm{~A}}$ group (Table I) revealed similar results to those of the anti-E7 test group. Highly similar results were reported between the two protein biomarkers.

ISH analysis of HPV E7 $m R N A$. In the present study, antibody-binding specificity to E7 antigen was investigated via IHC staining. In addition, HPV E7 mRNA expression levels were investigated via ISH using adjacent sections from the same tissue blocks used for E7 antibody staining by IHC. As presented in Fig. 5, E7 mAbs and E7 mRNA probes revealed similar localization of positively stained loci or regions on the slides, indicating the accurate recognition of the antibodies to the endogenous E7 antigen in the immunohistochemistry assay. Morphologically aberrant cells exhibited the typical punctate dots following the application of the E7 mRNA probe via ISH; staining intensity also appeared to be increased with the increasing degree of cancer. In this set of experiments, the mRNA and protein staining patterns appeared to be co-localized in the same areas in the lesions. Antibody 
staining indicated that the E7 mRNA expression levels were associated with the intensity and location of E7 protein expression. The findings of the present study further confirmed the specific immunoreaction of E7 antibodies and the endogenous tumor antigen E7 within cervical lesion tissue.

\section{Discussion}

Cervical cancer is a leading cause of cancer-associated mortality in females worldwide and the second most common cancer in females in developing countries (1). According to the data released by the National Central Cancer Registry of China, the incidence of cervical cancer in China was 15/100,000 in 2015, which is still listed as one of the major malignant tumors threatening female health in China (22). Fortunately, cervical cancer is preventable and curable at the early stages due to the relatively slow progression of cervical carcinoma (1). The morbidity and mortality of cervical cancer has been remarkably reduced by the early screening tests, including Pap-smear and liquid-based cytology (9). Low sensitivity and false positive results from the abnormal morphology of ASC-US are major limitations of cytology tests used in primary screening. According a meta-analysis of Pap-smear tests and LBC, the sensitivity and accuracy of cervical cytology exhibited wide variation in different regions and institutions (23). In addition, a study of a large population revealed that $30-60 \%$ of patients with invasive cervical cancer were reported to be normal in Pap-smear testing 3-5 years prior to diagnosis, indicating that the cervical cytology test has a poor negative predictive value (24). By contrast, the development of molecular HPV testing has largely improved the detection sensitivity, however, it is limited by its low overall specificity and low positive predictive value $(10,25)$. A desirable improvement in the early screening is to identify the real-time phenotype of precancerous cervical lesions, and to avoid over diagnosis and/or treatment of patients with transient HPV infection only, or ASC-US and a low-grade LSIL cervical lesion.

Biomarkers can potentially improve the diagnostic interpretation of cytology tests for early stage cervical cancer (11). Despite the benefit of decreasing inter-observer variations for the diagnosis of LSILs, the clinical significance of $\mathrm{p} 16^{\mathrm{INK} 4 \mathrm{~A}}$ is limited by its intrinsic properties, including the inconsistent expression in tumors, lack of tumor specificity and the lack of uniformity in scoring systems.

E7 is a pivotal oncoprotein, with expression required to maintain the transformed phenotype in hrHPV-induced human cancers. The conformational, structural and biochemical behavior of E7 protein has been extensively characterized (18). Several conformational E7 species have been identified, including a nuclear dimer and high molecular weight spherical oligomers in the cytoplasm of transformed cells (26). It has been hypothesized that the predominant species of E7 oligomers may exist in transformed cells, and contain epitopes that are not exposed in the dimer. The complexity of conformational changes in E7 protein made the 'rational design' and construction of an E7 antibody extremely difficult.

Considering its highly specific expression in transformed cervical cells, HPV E7 protein has been well reported as an appropriate biomarker candidate for the detection of cervical cancer (12). A number of studies reported the detection of E7 proteins using either polyclonal antibodies or mAbs (12,26-32). Lidqvist et al (18) described the production of mouse mAbs to HPV E7 protein and use for testing LBC slides. However, data from a clinical evaluation was not reported. Faoro et al (26) reported a new E7 antibody with limited antibody specificity data and indiscriminative immunoreactivity in LSIL and HSIL. Collectively, available reports have demonstrated a lack of thorough characterization of antibody specificity, or the evaluation of E7 protein as a biomarker for diagnostic testing.

The present study reported an empirical approach to develop several unique $\mathrm{mAbs}$ that recognize $\mathrm{E} 7$ proteins from HPV16, HPV18 as well as structurally-associated E7 proteins from other strains of hrHPVs. This may increase the range of diagnostic application for the analysis of clinical specimens. $\mathrm{mAb}$ were constructed using protein antigens of $\mathrm{E} 7$ from hrHPV16 and 18 and subsequently used to immunize mice and rabbits. $\mathrm{mAb}$ clones were screened and selected based on binding specificity to E7 proteins from numerous hrHPV strains, including HPV16 and 18, and structurally-associated hrHPV31, 33, 35, 39, 45, 52, 58, and 59. Antibody candidate clones demonstrating cross-reactivity with low risk strain HPV E7 proteins (HPV6 and 11) were excluded from subsequent analysis in the present study. Using a variety of characterization assays, it was confirmed that the selected mAbs specifically bind to the endogenous hrHPV E7 proteins in cervical cancer cells (CaSki and HeLa). Finally, the applications of these anti-E7 mAbs for the specific recognition of E7-expressing cervical cells in clinical specimens were demonstrated, via LBC slides and FFPE tissue slides by ICC or IHC, respectively. Of particular interest is investigation of the use of anti-E7 staining in ASC-US slides, which may contribute to the interpretation of cytology-based early screening tests. In addition, IHC analysis using the E7 antibody cocktail reagent produce similar results to $\mathrm{p} 16^{\mathrm{INK} 4 \mathrm{~A}}$ immunostaining, indicating the feasibility of using E7 as a specific biomarker for identification of premalignant cervical epithelial lesions.

In summary, the HPV E7 protein encoded by the viral genome, but only expressed in transformed human cells, may be considered as an ideal biomarker for the early detection of precancerous lesions. The detection of E7 protein expression may effectively distinguish malignant transformations from transient HPV infections. This E7 protein identification technique may be used in either ICC or IHC analyses of cervical samples, and provide more reliable results for improved efficiency in cervical cancer screening and early diagnosis.

\section{Acknowledgements}

The authors acknowledge the support and critical reading of this manuscript from Dr Jiliang $\mathrm{Hu}$ of the College of Pharmacology, Guangzhou University (Guangzhou, China).

\section{Funding}

No funding was received.

\section{Availability of data and materials}

The datasets used and/or analyzed during the current study are available from the corresponding author on reasonable request. 


\section{Authors' contributions}

LS and XC designed the experiments. LS, FH and CS performed the experiments. YH and YL analyzed the data. All authors read the manuscript.

\section{Ethics approval and consent to participate}

The use of human tissue in the present study was approved by the Institutional Review Board at the First Affiliated Hospital of Soochow University, prior to data collection. Written informed consent was obtained from all participants involved in this study.

\section{Consent for publication}

Not applicable.

\section{Competing interests}

The authors declare that they have no competing interests.

\section{References}

1. McGuire S: World Cancer Report 2014. Geneva, Switzerland: World Health Organization, International Agency for Research on Cancer, WHO Press, 2015. Adv Nutr 7: 418-419, 2016.

2. Crosbie EJ, Einstein MH, Franceschi S and Kitchener HC: Human papillomavirus and cervical cancer. Lancet 382: 889-899, 2013.

3. Choi YJ and Park JS: Clinical significance of human papillomavirus genotyping. J Gynecol Oncol 27: e21, 2016.

4. Groves IJ and Coleman N: Pathogenesis of human papillomavirus-associated mucosal disease. J Pathol 235: 527-538, 2015

5. Gravitt PE: The known unknowns of HPV natural history. J Clin Invest 121: 4593-4599, 2011.

6. Yugawa T and Kiyono T: Molecular mechanisms of cervical carcinogenesis by high-risk human papillomaviruses: Novel functions of E6 and E7 oncoproteins. Rev Med Virol 19: 97-113, 2009.

7. Rashid NN, Rothan HA and Yusoff MS: The association of mammalian DREAM complex and HPV16 E7 proteins. Am J Cancer Res 5: 3525-3533, 2015.

8. Egawa N, Egawa K, Griffin H and Doorbar J: Human papillomaviruses; epithelial tropisms, and the development of neoplasia. Viruses 7: 3863-3890, 2015.

9. Saslow D, Solomon D, Lawson HW, Killackey M, Kulasingam SL, Cain J, Garcia FA, Moriarty AT, Waxman AG, Wilbur DC, et al: American Cancer Society, American Society for Colposcopy and Cervical Pathology, and American Society for Clinical Pathology screening guidelines for the prevention and early detection of cervical cancer. Am J Clin Pathol 137: 516-542, 2012.

10. Goodman A: HPV testing as a screen for cervical cancer. BMJ 350: h2372, 2015.

11. Dasari S, Wudayagiri $\mathrm{R}$ and Valluru L: Cervical cancer: Biomarkers for diagnosis and treatment. Clin Chim Acta 445: 7-11, 2015.

12. Stiasny A, Kuhn C, Mayr D, Alexiou C, Janko C, Wiest I, Jeschke U and Kost B: Immunohistochemical evaluation of E6/E7 HPV oncoproteins staining in cervical cancer. Anticancer Res 36: 3195-3198, 2016.

13. Hoffmann A and Roeder RG: Purification of his-tagged proteins in non-denaturing conditions suggests a convenient method for protein interaction studies. Nucleic Acids Res 19: 6337-6338, 1991.
14. Harper S and Speicher DW: Purification of proteins fused to glutathione S-transferase. Methods Mol Biol 681: 259-280, 2011.

15. Morbini P, Alberizzi P, Tinelli C, Paglino C, Bertino G, Comoli P, Pedrazzoli $\mathrm{P}$ and Benazzo M: Identification of transcriptionally active HPV infection in formalin-fixed, paraffin-embedded biopsies of oropharyngeal carcinoma. Hum Pathol 46: 681-689, 2015.

16. Pirog EC: Immunohistochemistry and in situ hybridization for the diagnosis and classification of squamous lesions of the anogenital region. Semin Diagn Pathol 32: 409-418, 2015.

17. Szarewski A, Mesher D, Cadman L, Austin J, Ashdown-Barr L, Ho L, Terry G, Liddle S, Young M, Stoler M, et al: Comparison of seven tests for high-grade cervical intraepithelial neoplasia in women with abnomal smears: The predictors 2 study. J Clin Microbiol 50: 1867-1873, 2012.

18. Lidqvist M, Nilsson O, Holmgren J, Hölters S, Röijer E, Dürst M and Fermér C: Detection of human papillomavirus oncoprotein E7 in liquid-based cytology. J Gen Virol 93: 356-363, 2012.

19. Marks JD and Bradbury A: PCR cloning of human immunoglobulin genes. Methods Mol Biol 248: 117-134, 2004.

20. Solomon D, Davey D, Kurman R, Moriarty A, O'Connor D, Prey M, Raab S, Sherman M, Wilbur D, Wright T Jr, et al: The 2001 Bethesda System: Terminology for reporting results of cervical cytology. JAMA 287: 2114-2119, 2002.

21. Szalmás A, Tomaić V, Basukala O, Massimi P, Mittal S, Kónya J and Banks L: The PTPN14 tumor suppressor is a degradation target of human papillomavirus E7. J Virol 91: e00057-17, 2017.

22. Chen W, Zheng R, Baade PD, Zhang S, Zeng H, Bray F, Jemal A, Yu XQ and He J: Cancer statistics in China, 2015. CA Cancer J Clin 66: 115-132, 2016.

23. Nanda K, McCrory DC, Myers ER, Bastian LA, Hasselblad V, Hickey JD and Matchar DB: Accuracy of the Papanicolaou test in screening for and follow-up of cervical cytologic abnormalities: A systematic review. Ann Intern Med 132: 810-819, 2000.

24. Katki HA, Kinney WK, Fetterman B, Lorey T, Poitras NE, Cheung L, Demuth F, Schiffman M, Wacholder S and Castle PE: Cervical cancer risk for women undergoing concurrent testing for human papillomavirus and cervical cytology: A population-based study in routine clinical practice. Lancet Oncol 12: 663-672, 2011

25. Zazove P, Reed BD, Gregoire L, Ferenczy A, Gorenflo DW and Lancaster WD: Low false-negative rate of PCR analysis for detecting human papillomavirus-related cervical lesions. J Clin Microbiol 36: 2708-2713, 1998.

26. Faoro V, Barbazza R, Bonin S, Brunetti D, Sulfaro S and Stanta G: Detection of HPV E7 oncoviral protein in cervical lesions by a new antibody. Appl Immunohistochem Mol Morphol 21: 341-350, 2013.

27. Ramirez N, Guerra F, Camporeale G, Quintana S, Diaz LB, Cuneo N, Villacorta Hidalgo J, Tatti SA, Alonso LG, Borkosky SS, et al: Expressions of E2 and E7-HPV16 proteins in pre-malignant and malignant lesions of the uterine cervix. Biotech Histochem 90: 573-580, 2015.

28. Arbyn M, Paraskevaidis E, Martin-Hirsch P, Prendiville W and Dillner J: Clinical utility of HPV-DNA detection: Triage of minor cervical lesions, follow-up of women treated for high-grade CIN: An update of pooled evidence. Gynecol Oncol 99: (3 Suppl 1): S7-S11, 2005.

29. Nasiell K, Roger V and Nasiell M: Behavior of mild cervical dysplasia during long term follow-up. Obstet Gynecol 67: 665-669, 1986.

30. Holowaty P, Miller AB, Rohan T and To T: Natural history of dysplasia of the uterine cervix. J Natl Cancer Inst 91: 252-258, 1999.

31. van Bogaert LJ: P16INK4a immunocytochemistry/immunohistochemistry: Need for scoring uniformization to be clinically useful in gynecological pathology. Ann Diagn Pathol 16: 422-426, 2012.

32. Dantur K, Alonso L, Castaño E, Morelli L, Centeno-Crowley JM, Vighi S and de Prat-Gay G: Cytosolic accumulation of HPV16 E7 oligomers supports different transformation routes for the prototypic viral oncoprotein: The amyloid-cancer connection. Int J Cancer 125: 1902-1911, 2009. 